\title{
Awareness and Use of Caffeine by Athletes Competing at the 2005 Ironman Triathlon World Championships
}

\author{
Ben Desbrow and Michael Leveritt
}

\begin{abstract}
This study assessed the knowledge, prevalence, and quantity of caffeine use by athletes competing at the 2005 Ironman Triathlon World Championships. Caffeine-related questionnaires were self-administered to 140 (105 male and 35 female, $40.3 \pm 10.7$ y) athletes representing 16 countries. Fifty of these athletes further consented to immediate post-race blood samples for analysis of plasma caffeine and paraxanthine using high-performance liquid chromatography (HPLC). Seventy-two percent of 70 athletes correctly identified caffeine as being an unrestricted substance in triathlon. The majority of athletes [125 (89\%)] were planning on using a caffeinated substance immediately prior to or throughout the race. Cola drinks (78\%), caffeinated gels (42\%), coffee (usually pre-race) (37\%), energy drinks (13\%), and NoDoz tablets (9\%) were the most popular caffeinated choices. Mean \pm standard deviation (and range) post race plasma caffeine and paraxanthine levels were $22.3 \pm 20 \mu \mathrm{mol} / \mathrm{L}$ (1.7 to 98.4$)$ and $9.4 \pm 6 \mu \mathrm{mol} / \mathrm{L}(1.8$ to 28.9), respectively. Seven athletes (14\%) finished with plasma caffeine levels $\geq 40 \mu \mathrm{mol} / \mathrm{L}$. Plasma values from elite athletes did not differ from age group competitors. Despite the prevalence of its consumption and the training experience of this athletic group, over one quarter of athletes remained either confused or uninformed about caffeine's legality. Levels of plasma caffeine taken immediately post race indicated that athletes typically finish with quantities of caffeine that have been shown to improve endurance performance (i.e., $\approx 20 \mu \mathrm{mol} / \mathrm{L}$ or a dose of $\geq 3 \mathrm{mg} / \mathrm{kg}$ body weight).
\end{abstract}

Key Words: ergogenic aids, supplements, endurance performance

Caffeine is probably the most widely used pharmacologically active substance in the world. Although caffeine is found in a number of foods, it is most frequently consumed in coffee, tea, and cola beverages (11). In January 2004, the World Anti-Doping Agency (WADA) removed caffeine from its restricted substances list (18). Consequently, athletes are now able to consume caffeine in conjunction with WADA-sanctioned sports, without fear of doping code violations. However, caf-

Desbrow is with the School of Public Health and Heart Foundation Research Centre, Griffith University, Gold Coast, Queensland, Australia. Leveritt is with the School of Human Movement Studies, University of Queensland and the Centre of Excellence for Applied Sport Science Research, Queensland Academy of Sport, Queensland, Australia. 
feine remains on WADA's monitoring program, indicating that the drug is placed under heightened scrutiny for further tracking of trends in use and possible abuse for future prohibition list consideration (19).

A substance or method can be placed on the WADA prohibited list if it meets two of the three following criteria: 1) it has the potential to be performance enhancing; 2) it represents an actual or potential risk to the athlete's health; and 3) it violates the spirit of sport (20). Therefore any decision concerning the prohibition status of caffeine requires considerable monitoring and expert debate.

The issue of caffeine's safety in endurance sport deserves consideration. Recently, Nawrot and colleagues (13) concluded that caffeine had no adverse effects at levels of up to $400 \mathrm{mg} / \mathrm{d}$ in healthy adults. A recent meta-analysis of the impact of coffee and caffeine on blood pressure indicated a dose-response effect. Trials containing $>410 \mathrm{mg} / \mathrm{d}$ of caffeine produced a significantly greater elevation in systolic blood pressure when compared to trials with lower caffeine doses (14). It is important to remember that both of these findings originate and are orientated towards the free-living population rather than people competing in endurance triathlons.

To date the majority of research performed in the area of caffeine in sport has focused on its potential ergogenic properties. More than 60 original studies have been published in peer-reviewed journals documenting caffeine's effect on various aspects of sporting performance (for review, see reference 2). In a recent meta-analysis of controlled trials, caffeine was confirmed as ergogenic, particularly for endurance sport (3). Furthermore, this performance enhancement was demonstrated at levels that are not likely to produce acute or chronic health problems (3 to $6 \mathrm{mg} / \mathrm{kg}$ body weight) $(2,13)$.

Despite this scientific interest in caffeine's ergogenic effects there remains a lack of ultra endurance caffeine performance data. No published study has investigated the impact of caffeine on Ironman triathlon performance. Furthermore, there are no studies that have investigated the effect of caffeine on exercise performance over the duration or distance of one of the individual disciplines required to complete an Ironman triathlon (i.e., $3.8 \mathrm{~km}$ swim, $180 \mathrm{~km}$ cycle, or $42.2 \mathrm{~km}$ run).

Limited information exists on the recent use of caffeine by athletes. Graham (5) stated that in 1993, 27\% of Canadian teenagers surveyed reported using caffeine to improve their sports performance. In 2003, Slater and co-workers (14) also demonstrated a high prevalence of caffeine use (37\%) in Singaporean athletes. One year later, lower rates of caffeine consumption $11 \%$ (mostly by college wrestlers) were reported by researchers in the US (4). These lower rates were confirmed by a recent study reporting that despite caffeine having the highest usage of all dietary supplements it was rarely consumed by athletes for its ergogenic benefits (rather for pleasure and alertness) (10).

Three issues impact on the relevance of previous studies when assessing the current caffeine behaviors of athletes. First, the majority of these studies (4, $5,15)$ were conducted prior to January 1,2004 , when there is the potential that under-reporting by athletes concerned by the possible legal implications of their responses has influenced the research findings. Second, all of these studies rely on self-reported information and have not had the reported behaviors confirmed by any objective measurements (e.g., plasma/urinary caffeine concentrations). Finally, many of the studies reporting on caffeine behaviors in athletes have assessed "usual" 
total dietary or supplement behaviors, rather than the prevalence of caffeine taken in conjunction with competition to specifically attempt to elicit a performance enhancement $(4,10,15)$.

The only published study quoting urinary caffeine levels in athletes since caffeine's removal from the prohibited substances list does not include data collected after 2002 (17). Unpublished information available from WADA indicates some changes in the number of high caffeine results recorded by elite athletes, $(0.035 \%$ positive tests in $2002(n=70,214)$ versus $0.152 \%$ positive tests in 2004 $(n=18,394))$ (personal communication, WADA, November 25, 2005). Importantly, this data only describes tests that have recorded urinary caffeine concentrations of $>12 \mu \mathrm{g} / \mathrm{mL}$ (i.e., a former positive test) and does not provide any information on the prevalence of caffeine use at lower doses. In addition, the total number of caffeine tests (and testing centers) has fallen considerably since removal from the prohibited substances list. As a consequence, objective information available on the caffeine behaviors of elite athletes has decreased despite the likelihood that more athletes could experiment with caffeine since its removal from the WADA prohibited substances list.

The aim of this study was to investigate the knowledge and use of caffeine within a group of athletes competing at a major international triathlon. Professional athletes competing at this event are sanctioned in accordance with the WADA anti-doping code. Results will provide information concerning the levels of caffeine used in a group of well-trained endurance athletes (both elite and age group competitors) 22 months following caffeine's legalization. Planned caffeine use will be confirmed by an objective measure (plasma caffeine). This information will confirm the accuracy of the self-reported caffeine intake data. It is anticipated that a cross-sectional investigation into the caffeine intake behaviors of athletes will improve the advice and education provided to these athletes regarding safe caffeine practices and will inform doping control agencies regarding caffeine's current use as a legal ergogenic aid.

\section{Methods}

\section{Event Description}

The 2005 Hawaiian Ironman Traithlon World Championship was operated under license from the World Triathlon Corporation (WTC). The WTC Ironman races are not sanctioned by the International Triathlon Union and are therefore not an official triathlon world championship. The event consists of a $3.8 \mathrm{~km}$ swim, 180 $\mathrm{km}$ cycle, and a $42.2 \mathrm{~km}$ run. Athletes qualify for the event and participate as open competitors (professional) or compete within age groups.

\section{Study Subjects}

Caffeine-related questionnaires were self-administered to 141 randomly selected athletes immediately following race registration. One questionnaire was returned incomplete. Information was therefore collected on 140 [105 males, age $41.1 \pm$ 11.3 y (mean \pm standard deviation), body weight $75.1 \pm 8.2 \mathrm{~kg}$ and 35 females, age $37.9 \pm 7.8$ y, body weight $57.9 \pm 5.4 \mathrm{~kg}$ ] athletes ( $8 \%$ of all competitors) from 16 countries (see Figure 1). 


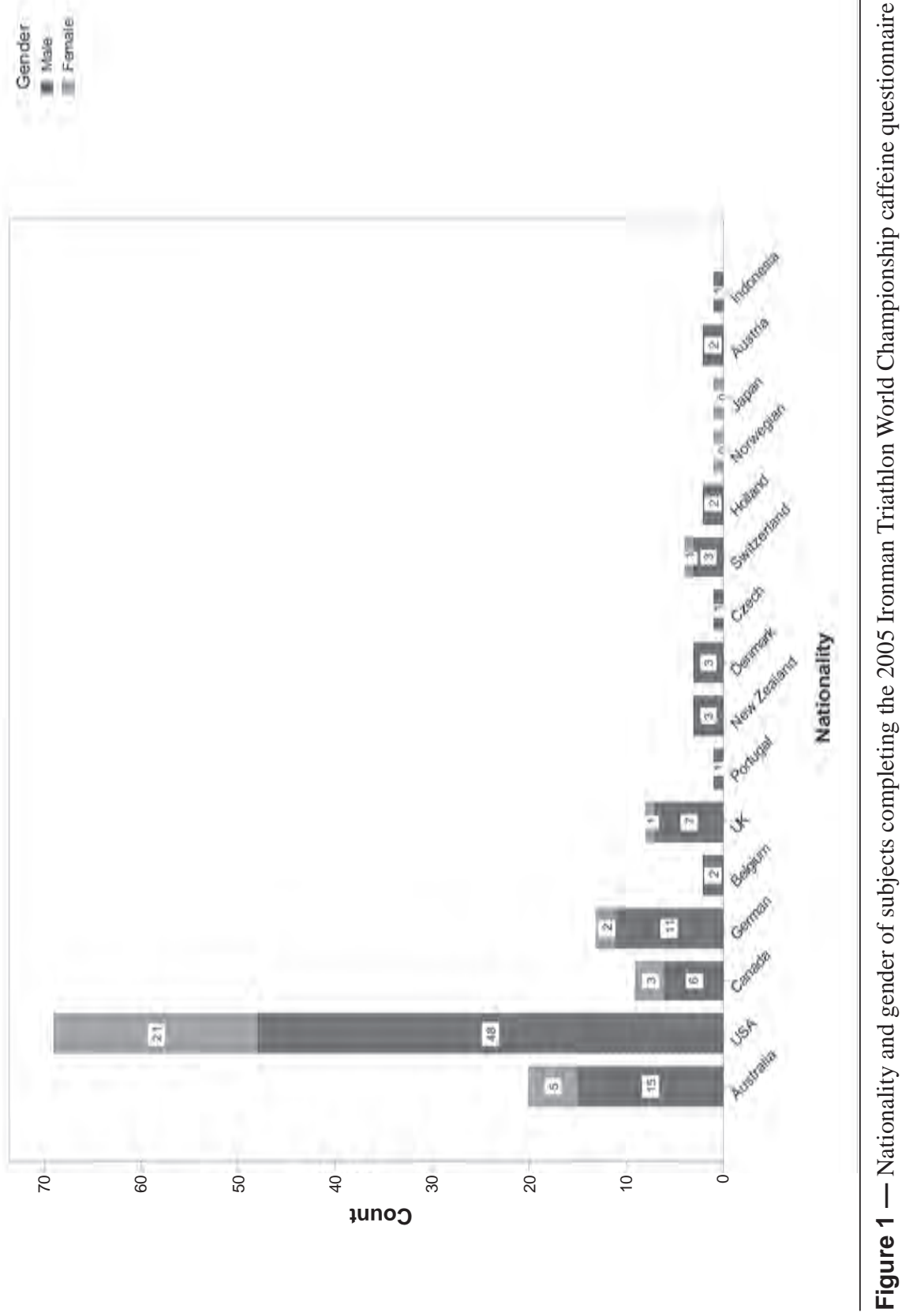


Fifty of these athletes ( 34 males, age $40.0 \pm 11.1 \mathrm{y}$, body weight $74.3 \pm 6.7$ $\mathrm{kg}$ and 16 females, age $38.0 \pm 8.0 \mathrm{y}$, body weight $59.0 \pm 6.3 \mathrm{~kg}$ ) further consented to providing immediate post-race blood samples for analysis of plasma caffeine and paraxanthine. Subjects in both components of the research were a mixture of elite and age-group competitors. Elite athletes were defined as those registered as professional triathletes or those finishing in the top three places of age categories $\leq 45 \mathrm{y}$. The term age-group competitor is used to describe the remaining very well trained endurance athletes that compete in this event. Figure 2 displays race times relative to the subject's ability classification. The questionnaire was only available in English, consequently limiting the potential diversity of research subjects. The study was reviewed and approved by the Griffith University Human Research Ethics Committee.

\section{Questionnaire Design}

A self-administered questionnaire investigating subject's caffeine knowledge, attitudes, use history, planned consumption, and caffeine-related side effects was developed from a previous caffeine use questionnaire that had been pilot tested on athletes by members of the research team (Table 1) (Note: Only data related to knowledge and planned consumption is reported in this study).

The questionnaire initially assessed the subjects intent to use caffeinated products throughout the event (caffeine users versus non-users). Separate components of the remaining questionnaire were completed based on this intention.

Caffeine Non-Users: History of previous caffeine use and rationale for not using caffeine were investigated (open-ended question).

Caffeine Users: Subjects outlined the type, timing, and approximate amount of caffeinated substance that they intended on consuming throughout the race. The history of use was also investigated by noting the length of experience with using caffeine and by offering the athlete the opportunity to provide an example (open-ended question) of either a positive or negative experience when using caffeine in the past.

\section{Plasma Caffeine and Paraxanthine Collection}

Consenting athletes $(n=50)$ were identified at the finish line by research assistants and escorted directly to the medical recovery area. On presentation (approximately $10 \mathrm{~min}$ post-race, personal observation) $5 \mathrm{~mL}$ of blood was collected from the antecubital vein into lithium heparin sample tubes. The samples were then immediately centrifuged at $3300 \mathrm{rpm}$ for 10 mins (Horizon miniE, The Drucker Co., Philipsburg, PA). The separated plasma was then frozen for subsequent caffeine and paraxanthine analysis. During the blood sampling procedure subjects were asked to confirm or modify their planned caffeine intake to indicate actual consumption.

\section{Plasma Caffeine and Paraxanthine Analysis}

Quantification of plasma caffeine and paraxanthine was performed using highperformance liquid chromatography (HPLC) via a method adapted from the validated technique described by Koch et al. (9). Specific details of the analysis are described below. 


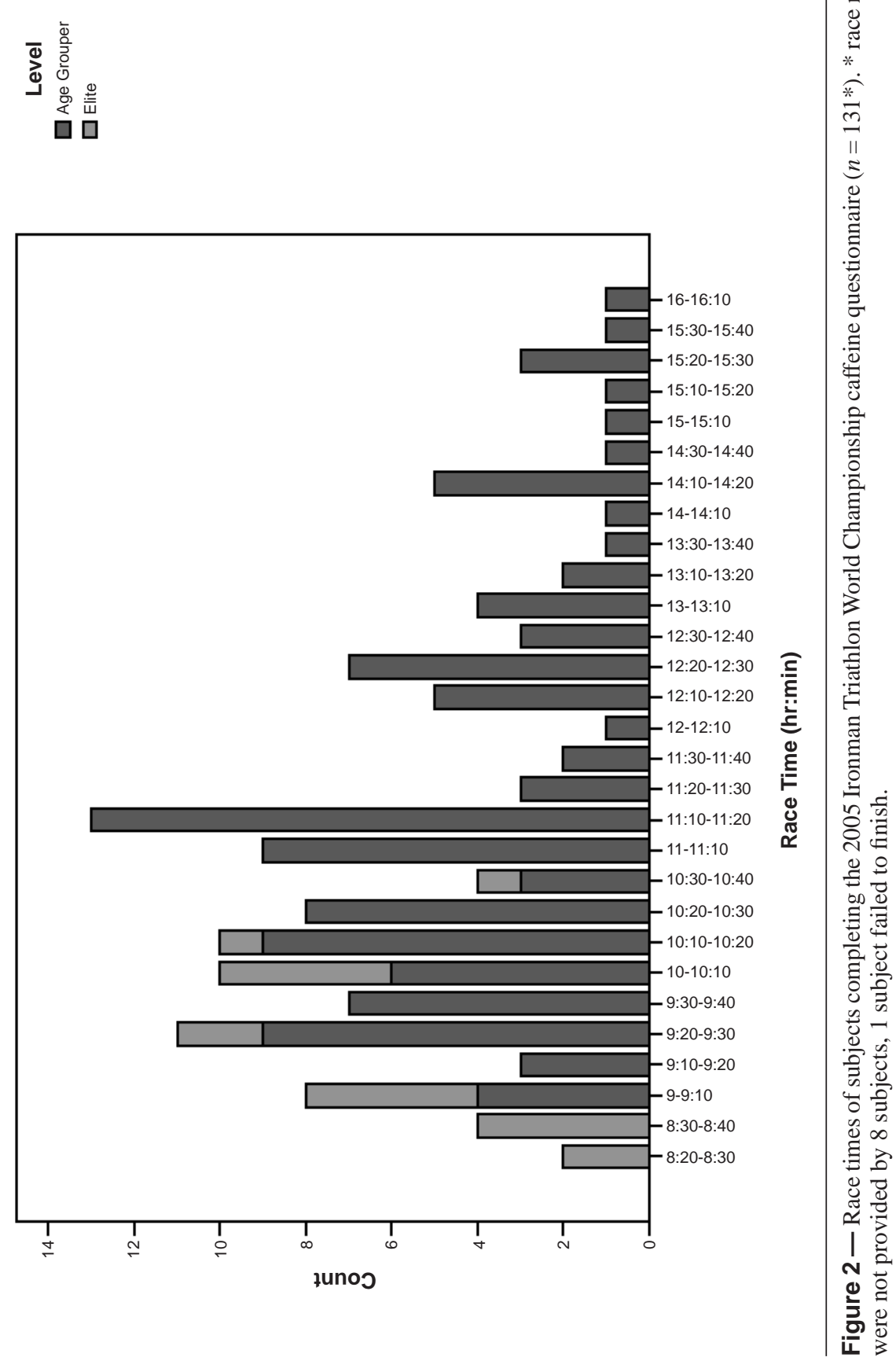




\section{Table 1 Self-Administered Questionnaire Components}

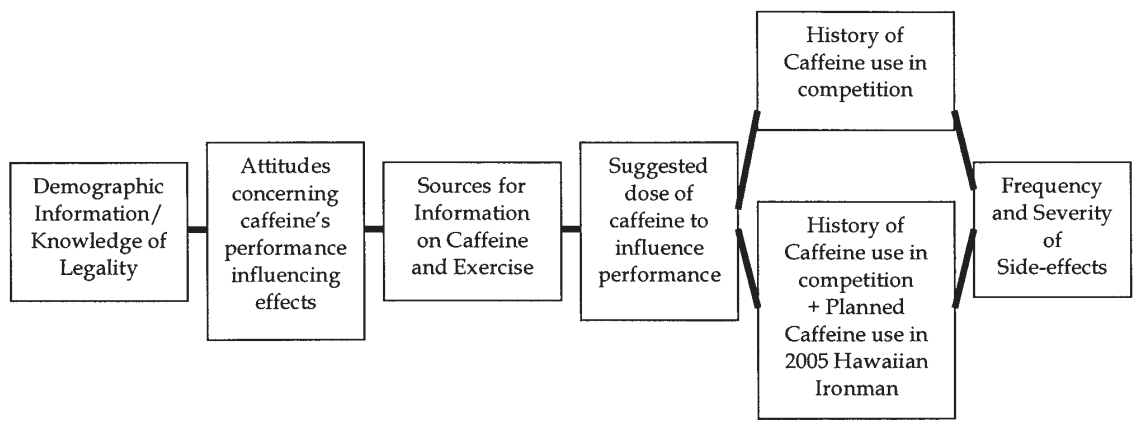

(Nb. Only data related to knowledge and planned consumption is reported).

Equipment/Materials. The method of analysis was performed using the following HPLC system: pump, Waters M45; UV detection, Applied Biosystems 757, with detection wavelength set to $273 \mathrm{~nm}$; integrator, Shimadzu-CR3A; column, LiChrospher $5 \mu \mathrm{m}$ RP18 $(25 \mathrm{~cm} \times 4.6 \mathrm{~mm})$; and injector, Rheodyne 7125 . The $\mathrm{pH}$ of the mobile phase was adjusted using a Radiometer Copenhagen PHM83 Autocal and filtrated over a Millipore GVHP04700 filter (pore size, $0.22 \mathrm{~mm}$ ).

Mobile Phase. The mobile phase used in this assay was composed of $830 \mathrm{~mL} 0.05$ $\mathrm{M} \mathrm{NaH} 2 \mathrm{PO} 4,300 \mathrm{~mL}$ triethylamine (pH 6.4) and $280 \mathrm{~mL}$ methanol. The eluent was filtrated over a Millipore filter (Supelco $0.2 \mu \mathrm{m} \times 47 \mathrm{~mm}$, Nylon 66 membranes) before it was used in the assay. The flow of the eluent was $1.5 \mathrm{~mL} / \mathrm{min}$.

Preparation of Standard and Validation Solutions. A stock solution of 2000 $\mu \mathrm{mol} / \mathrm{L}$ caffeine and $500 \mu \mathrm{mol} / \mathrm{L}$ paraxanthine was used for the preparation of standard solutions of caffeine and paraxanthine. All standard solutions (25, 50, and $100 \mu \mathrm{mol} / \mathrm{L}$ ) were prepared by diluting this stock solution.

Sample Preparation. Serum proteins were precipitated before the sample could be injected onto the chromatographic system. Protein precipitation was achieved using the following method: $50 \mu \mathrm{L}$ serum $+100 \mu \mathrm{L}$ methanol were mixed on a Vortex for $1 \mathrm{~min}$ and centrifuged at $12,000 \mathrm{rpm}$ for $10 \mathrm{~min}$. Next, $25 \mu \mathrm{L}$ of supernatant were injected into the HPLC system.

Quantification. Quantification of caffeine and paraxanthine was achieved by comparing peak areas to those of standards.

\section{Quantification of Planned and Actual Caffeine Consumption}

An accredited sports dietitian calculated the planned and actual caffeine intakes of subjects based on reported food and beverage intake using a summary table of caffeine values (1). For standardization of intake data, the following three assumptions were made if no further information was provided by the subject: 1) all drinks provided at aid stations were $100 \mathrm{~mL}, 2$ ) carbohydrate gels did not contain caffeine and (when uncertain) caffeinated carbohydrate gels provided $25 \mathrm{mg}$ of caffeine/sachet, and 3) No-Doz tablets each contained $200 \mathrm{mg}$ of caffeine (as per 
standard US dose). Reported planned caffeine intakes are expressed as mg (total) and $\mathrm{mg} / \mathrm{kg}$ body weight (relative) values.

\section{Statistical Analysis}

All data was coded and entered into SPSS software (SPSS, Inc. version 12.0.1, Chicago, IL) for analysis. Descriptive data analysis (mean, standard deviation, ranges, and percentages) was performed. All data is expressed as means \pm standard deviation unless otherwise indicated. All percentages are expressed as numbers relative to the total that completed the relevant questionnaire sections. Correlation coefficients for relative actual caffeine intake and plasma caffeine and plasma paraxanthine were calculated, as was the correlation between planned and actual reported caffeine intake. An independent samples $t$-test was conducted to determine if knowledge of prohibition status affected mean actual caffeine intake. One-way ANOVA was applied to assess if mean caffeine (both actual consumption and plasma) values differed relative to an athlete's nationality. The chi-square calculation was performed to investigate differences between knowledge and level of ability. Statistical significance was set at $P \leq 0.05$.

\section{Results}

\section{Caffeine Knowledge}

One hundred (72\%) athletes correctly identified caffeine as being unrestricted in triathlon. Figure 3 illustrates the relative responses to the question regarding caffeine's prohibition status. The elite category $(n=18)$ consists of 12 athletes registered as professionals and 6 podium-placed age category finishers. There were no differences between elite and age-group athletes regarding knowledge of caffeine's legality $\left(\chi^{2}=2.75, P=0.097\right)$.

\section{Planned Caffeine Use}

One hundred and twenty-five (89\%) athletes were reportedly planning on using a caffeinated substance immediately prior to or throughout the race. Cola beverages (78\%), caffeinated gels (42\%), coffee (usually pre-race) (37\%), energy drinks (13\%), and No-Doz tablets (9\%) were the most popular caffeinated choices. Due to incomplete data, only 93 athletes could have their planned caffeine intake accurately estimated. Mean (range) planned intake and mean (range) planned relative intake were 300 ( 0 to 1780 ) $\mathrm{mg}$ and 4.4 ( 0 to 23.7 ) $\mathrm{mg} / \mathrm{kg}$ body weight, respectively. Planned caffeine was highly correlated to actual intake $(R=0.80, P<0.01)$. Elite athletes planned to consume more caffeine than age group competitors $(476 \pm 298$ $\mathrm{mg}$ versus $279 \pm 289 \mathrm{mg}, P=0.044$ ) however this relationship was not evident when comparing planned relative doses. The influence of an athlete's knowledge regarding caffeine's prohibition status and the athlete's planned caffeine intake approached statistical significance $(P=0.079)$ with higher caffeine doses planned by athletes with the greater awareness of caffeine's legality (337 $\pm 322 \mathrm{mg}$ versus $219 \pm 189 \mathrm{mg}$ ). No differences were observed between planned (either total or relative) caffeine intake and age, gender, or nationality. 


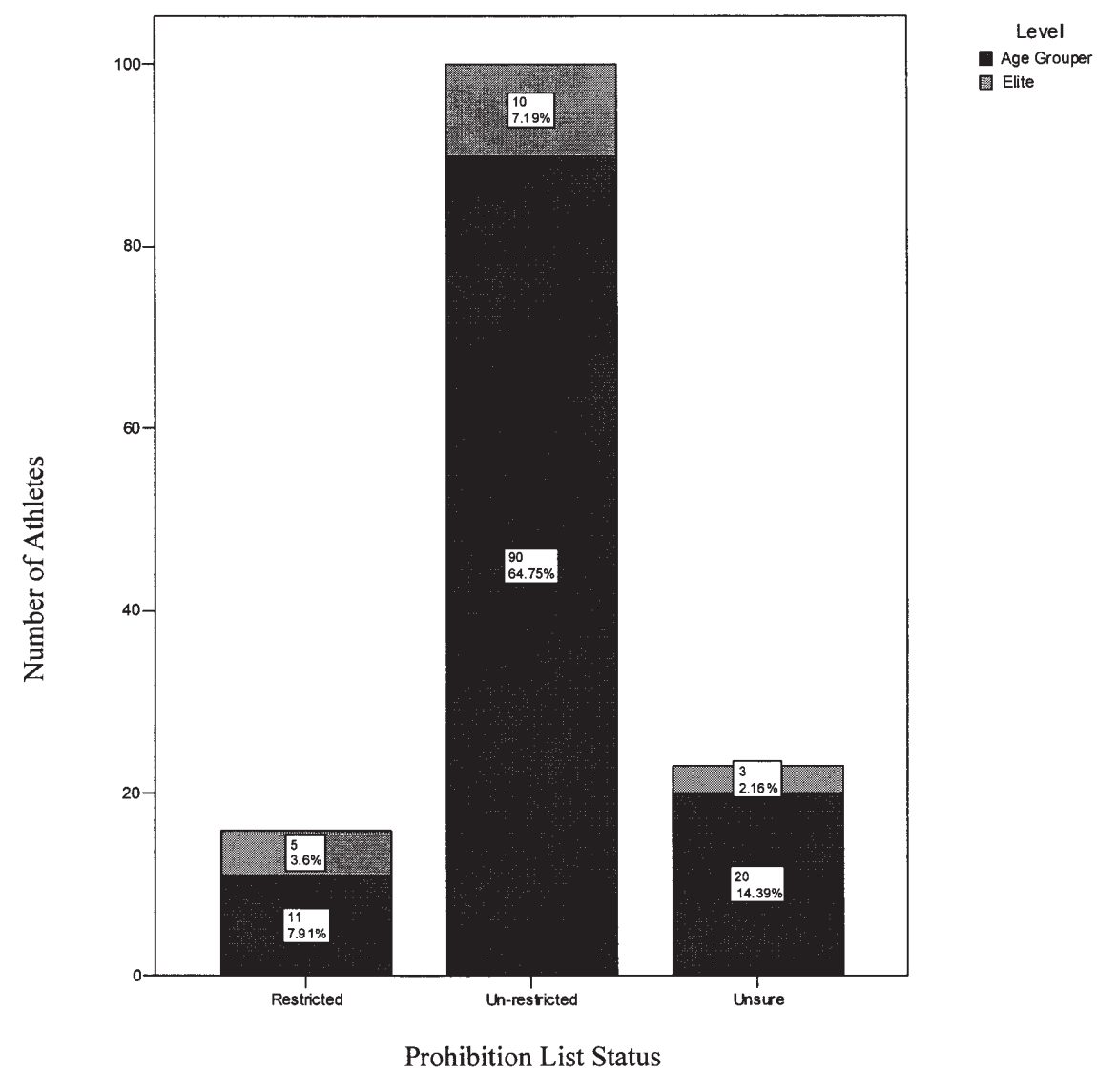

Figure 3 - Knowledge of caffeine's prohibition status by subjects competing in the 2005 Ironman World Championship $(n=139)$.

\section{Actual Caffeine Use}

Forty-eight athletes were able to provide information regarding their actual caffeine intake on completion of the race. Table 2 provides a summary of reported actual caffeine consumption. There were no differences reported between elite and age group competitors regarding actual caffeine consumption. Cola beverages were commonly consumed both during the bike and run disciplines $(22 \%)$ as well as on the run exclusively (60\%), whereas approximately half of those using No-Doz tablets ingested them during both the bike and cycle legs as opposed to the run exclusively (25\%).

Table 3 displays data relating to the influence of awareness of caffeine's prohibition status on actual caffeine consumption. Results indicated that those athletes with an awareness of caffeine's prohibition status were more likely to consume larger doses (both total; $P=0.23)$ and relative to body weight $(P=0.029)$ of caffeine than 
Table 2 Reported Actual Caffeine Intake Collected Immediately Following the 2005 Hawaiian Ironman World Championships: Comparison of Elite Versus Age Group Competitors

\begin{tabular}{lccc}
\hline & $\begin{array}{c}\text { All athletes } \\
(n=48)\end{array}$ & $\begin{array}{c}\text { Elite } \\
(n=10)\end{array}$ & $\begin{array}{c}\text { Age group } \\
(n=38)\end{array}$ \\
\hline $\begin{array}{l}\text { Total caffeine }(\mathrm{mg}) \\
\begin{array}{l}\text { Relative caffeine } \\
\text { (mg/kg body weight) }\end{array}\end{array}$ & $351 \pm 281$ & $380 \pm 296$ & $343 \pm 287$ \\
\hline
\end{tabular}

Values are means \pm standard deviation.

Table 3 Reported Actual Caffeine Intake Collected Immediately Following the 2005 Hawaiian Ironman World Championships: Influence of Knowledge Concerning Caffeine's Prohibition Status

\begin{tabular}{lccc}
\hline & $\begin{array}{c}\text { All athletes } \\
(n=48)\end{array}$ & $\begin{array}{c}\text { Unaware of } \\
\text { caffeine status } \\
(n=16)\end{array}$ & $\begin{array}{c}\text { Aware of } \\
\text { caffeine status } \\
(n=32)\end{array}$ \\
\hline $\begin{array}{l}\text { Total caffeine }(\mathrm{mg}) \\
\begin{array}{l}\text { Relative caffeine } \\
(\mathrm{mg} / \mathrm{kg} \text { body weight })\end{array}\end{array}$ & $351 \pm 281$ & $222 \pm 155$ & $415 \pm 309^{\mathrm{a}}$ \\
\hline
\end{tabular}

Values are means \pm standard deviation.

aindicates significant difference between actual total caffeine intakes based on an athlete's awareness of caffeine's prohibition list status $(P=0.023)$.

bindicates significant difference between actual relative caffeine intakes based on an athlete's awareness of caffeine's prohibition list status $(P=0.029)$.

those less familiar with its legality. No differences were observed between actual (either total or relative) caffeine intake and age, gender, or nationality.

\section{Plasma Caffeine and Paraxanthine}

Mean \pm standard deviation (range) post-race plasma caffeine and paraxanthine levels were $22.3 \pm 20 \mu \mathrm{mol} / \mathrm{L}$ (1.7 to 98.4 ) and $9.4 \pm 6 \mu \mathrm{mol} / \mathrm{L}$ (1.8 to 28.9 ), respectively. All athletes finished the race with detectable amounts of circulating caffeine. Seven athletes (14\%) finished with plasma caffeine levels $\geq 40 \mu \mathrm{mol} / \mathrm{L}$. Figure 4 illustrates the correlation between post-race plasma caffeine and reported actual relative caffeine intake $(R=0.69, P<0.01)$. This indicates that reported caffeine intakes correlate strongly with plasma levels. Plasma values from elite athletes did not differ from age group competitors.

\section{Discussion}

To date there are no published papers documenting the knowledge and behaviors of athletes regarding caffeine use in competition since its removal from the WADA anti- 


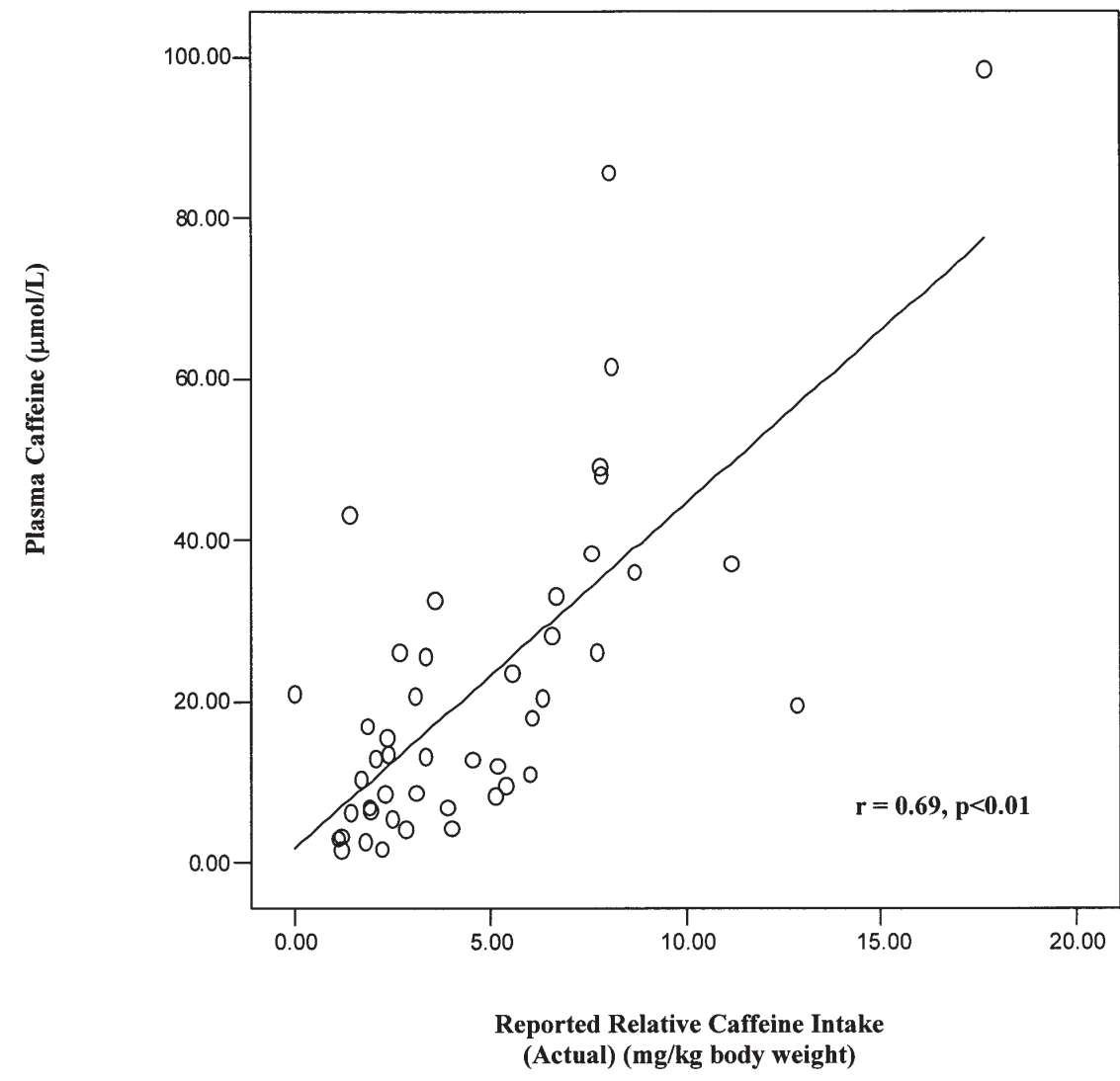

Figure 4 - Reported relative caffeine intake (actual) ( $\mathrm{mg} / \mathrm{kg}$ body weight) compared to post-race plasma caffeine $(\mu \mathrm{mol} / \mathrm{L})(n=50)$.

doping code. Such information would allow any conclusions regarding caffeine's place in sport to be based on evidence rather than anecdote.

One of the primary findings of this study was that detectable levels of caffeine were found in every sample of post-race blood collected from athletes $(n=50)$ completing the 2005 Hawaiian Ironman Triathlon. These samples were collected from a broad cross-section of well-trained endurance athletes (elite and age-group competitors). Given that peak plasma concentrations occur approximately $1.5 \mathrm{~h}$ after ingestion and that the plasma half-life for caffeine is approximately $7 \mathrm{~h} \mathrm{(12),}$ it appears evident that these athletes have typically ingested caffeine in moderate doses over a wide time period.

Laboratory studies indicate that a $1 \mathrm{~h}$ pre-exercise dose of caffeine required to elicit an average plasma caffeine level of approximately $22 \mu \mathrm{mol} / \mathrm{L}$ would need to be $\geq 3 \mathrm{mg} / \mathrm{kg}$ body weight (7). The present mean plasma paraxanthine values indicate sustained activity of the cytochrome P-450 1A2 enzyme (the major degrative pathway of caffeine) and are similar to those levels seen 4 to $8 \mathrm{~h}$ following 6 
$\mathrm{mg} / \mathrm{kg}$ body weight doses of caffeine $(8,12)$. Clearly, additional plasma caffeine and paraxanthine samples are required to confirm the precise timing and magnitude of peak plasma caffeine and paraxanthine levels achieved during events of this nature especially considering an average race time of approximately $11 \mathrm{~h}$ for our subjects. Despite this, the plasma data does support the reported information collected from athletes immediately post race indicating a mean caffeine consumption of $>4.5$ $\mathrm{mg} / \mathrm{kg}$ body weight either prior to or throughout the race.

Analysis of the relationship between the plasma caffeine data and the selfreported caffeine intakes indicated a significant positive correlation. This relationship suggests that self-reports of caffeine intake are a useful method of collecting caffeine dose information during endurance events.

No post-race urinary caffeine measures were collected during this study, therefore it is not possible to know the exact number of athletes that would have produced urinary levels greater than the previous WADA cut-off limit $(12 \mu \mathrm{g} / \mathrm{mL})$ for caffeine. Despite this, it is apparent that the majority of athletes competing in this event use caffeine at doses well below what has previously been reported to elicit a positive urinary test (i.e., $9 \mathrm{mg} / \mathrm{kg}$ ) (6). In addition, of the five athletes that reported planning to use $>9 \mathrm{mg} / \mathrm{kg}$ body weight of caffeine and subsequently had their planned intake verified immediately after the race, only two managed to achieve the planned intake. The present data demonstrates the tendency for athletes to consume doses of caffeine that are unlikely to result in urinary values above the former cut-off limit. It also illustrates the importance of the post-race follow-up to ensure the accuracy of information collected in research of this nature.

The safety aspects of taking higher doses of caffeine during endurance sport are controversial and it was not the intent of this investigation to clarify caffeine's involvement (if any) in the adverse events that occurred throughout the race. The present data indicate a mean caffeine intake of $351 \mathrm{mg}$ (range 0 to $1325 \mathrm{mg}$ ). Approximately $30 \%$ of athletes either planned to or actually did consume more than $400 \mathrm{mg}$ of caffeine in association with this event and the highest plasma values were associated with the ingestion of caffeine tablets (i.e., No-Doz tablets). It is important to consider, however, that any ergogenic benefits from caffeine are likely to be achieved at doses below or similar to the reported no adverse effect level (2).

Another key finding was the high prevalence of caffeine use despite a somewhat limited awareness from both elite and age-group athletes of the recent removal of caffeine from the prohibited substances list. The lack of difference in awareness regarding caffeine's legality between the elite and age-group athletes may, in part, be explained by the nature of "elite" competition in Ironman triathlon racing. The majority of athletes defined as elite for the purposes of this study are unlikely to be considered as national elite triathletes that compete over the traditional Olympic distance and are subsequently subjected to WADA testing. The lack of exposure by the elite athletes in the present study to national sports drug education programs and regular anti-doping testing limits the applicability of the present findings to the behaviors of the broader elite triathlete population.

The athlete's awareness of caffeine's legality was associated with their actual caffeine intake. This suggests that, not only are many athletes taking caffeine throughout this event, but that the dose of caffeine used is likely to increase if an athlete is aware of caffeine's position as an unrestricted substance. In addition, the demonstration that an athlete's awareness influences their actual caffeine intake 
suggests that the consumption of caffeine throughout endurance events of this nature is altering from one of incidental consumption as seen in previous dietary intake studies (16) to one of targeted supplementation. Certainly the $12(8.5 \%)$ athletes that planned to use No-Doz tablets throughout the race can be considered as doing nothing else. Clearly, caffeine is being used as a legal ergogenic aid and this has implications for coaches, race organizers, and sports medical/scientific staff. Likewise, there is a clear rationale to continue the monitoring of caffeine use by international anti-doping agencies.

The notion that all caffeine users are deliberately targeting caffeine for its performance enhancing properties is not without limitation. The present study uncovered one athlete that stated no caffeine had been consumed but recorded a substantial plasma caffeine concentration $(\approx 20 \mu \mathrm{mol} / \mathrm{L})$. Similarly, a number of athletes reported having not consumed caffeine containing foods/fluids but on additional questioning reported large intakes of cola beverages. Evidently, some athletes either do not have knowledge of which foods/fluids contain caffeine or they do not consider the consumption of them as "using" caffeine. There is also a possibility that athletes, who were not aware that caffeine is now unrestricted, are fearful of the repercussions of reporting "illegal" caffeine use. Consequently, care must be taken with the interpretation of reported caffeine intake data.

Several other limitations exist regarding the generalizability of the present data to the entire subject population. The sample size for the questionnaire $(n=$ 140) represents only $8 \%$ of those that competed. In addition, the possibility that a sampling bias towards those interested in caffeine or aware of caffeine's unrestricted status can not be entirely eliminated.

Nevertheless, this study demonstrated the nature of caffeine use in a group of well-trained endurance athletes. The present data indicated that athletes competing in the 2005 Hawaiian Ironman Triathlon often finish with quantities of caffeine that have been shown to improve endurance performance. Surprisingly, despite the prevalence of its consumption and the training experience of this athletic group over one quarter of athletes remained either confused or misinformed as to caffeine's legality. An understanding of the awareness and usage patterns of caffeine by athletes will contribute to the development of more effective education programs and resources for athletes on caffeine use in sport. Equally, by continuing to monitor the caffeine practice behaviors of athletes, decisions regarding the future prohibition list status of caffeine will be based on contemporary scientific evidence.

\section{Acknowledgments}

The generous help of Dr. Luke Haseler, Laura Dierenfield, Dr. Douglas Hiller, Penny Mack, and Gary Wilson is gratefully acknowledged. The editorial input of Assoc. Prof. Roger Hughes and Dr. Clare Minahan is recognized and appreciated. This study was funded by the Heart Foundation Research Centre, Griffith University.

\section{References}

1. Australian Sports Commission, Australian Institute of Sport-Sports Supplement Program Factsheet: Caffeine, Australian Government. www.ais.org.au/nutrition. Accessed February 7, 2006. 
2. Burke L., M. Cort, G. Cox, R. Crawford, B. Desbrow, M. Minehan et al. Dietary supplements and nutritional ergogenic aids in sport. In: Clinical Sports Nutrition, 3d ed., L. Burke \& V. Deakin (eds.) Sydney: McGraw Hill, 2006.

3. Doherty, M., and P. Smith. Effects of caffeine ingestion on exercise testing: a metaanalysis. Int. J. Sport Nutr. Exerc. Metab. 14:626-646, 2004.

4. Froiland, K., W. Koszewski, J. Hingst, and L. Kopecky. Nutritional supplement use among college athletes and their sources of information. Int. J. Sport Nutr. Exerc. Metab. 14:104-120, 2004.

5. Graham, T. Caffeine and exercise: metabolism, endurance and performance. Sports Med. 31:785-807, 2001.

6. Graham, T., and L. Spreit. Performance and metabolic responses to a high caffeine dose during prolonged exercise. J. App. Physiol. 71: 2292-2298, 1991.

7. Graham, T., and L. Spreit. Metabolic, catecholamine, and exercise performance responses to various doses of caffeine. J. App. Physiol. 78: 867-874, 1995.

8. Hunter, A., A.S.C. Gibson, M. Collins, M. Lambert, and T. Noakes. Caffeine ingestion does not alter performance during a 100-km cycling time trial. Int. J. Sport Nutr. Exerc. Metab. 12:438-452, 2002.

9. Koch, J., G.W. ten Tusscher, J. Kopple, and H. Guchelaar. Validation of a high-performance liquid chromatography assay for quantification of caffeine and paraxanthine in human serum in the context of CYP1A2 phenotyping. Biomed. Chrom. 13:309-314, 1999.

10. Kristiansen, M., R. Levy-Milne, S. Barr, and A. Flint. Dietary supplement use by varsity athletes at a Canadian university. Int. J. Sport Nutr. Exerc. Metab. 15:195-210, 2005.

11. Mandel, H. Update on caffeine consumption, disposition and action. Food Chem. Toxic. 40:1231-1234, 2002.

12. McLean, C., and T. Graham. Effects of exercise and thermal stress on caffeine pharmacokinetics in men and eumenorrheic women. J. App. Physiol. 93:1471-1478, 2002.

13. Nawrot, P., S. Jordan, J. Eastwood, J. Rotstein, A. Hugenholtz, and M. Feeley. Effects of caffeine on human health. Food Addit. Contam. 20:1-30, 2003.

14. Noordzij, M., C. Uiterwaal, L. Arends, F. Kok, D. Grobbee, and J. Geleijnse. Blood pressure response to chronic intake of coffee and caffeine: a meta-analysis of randomized control trials. J. Hypertension, 23:921-928, 2005.

15. Slater, G., B. Tan, and K.C. Teh. Dietary supplementation practices of Singaporean athletes. Int. J. Sport Nutr. Exerc. Metab. 13:320-332, 2003.

16. Speedy, D., T. Noakes, N. Kimber, I. Rogers, J. Thompson, D. Boswell, et al. Fluid balance during and after an ironman triathlon. Clin. J. Sports Med. 11:44-50, 2001.

17. Van Thuyne, W., K. Roels, and F.T. Delbeke. Distribution of caffeine levels in urine in different sports in relation to doping control. Int. J. Sports Med. 26:714-718, 2005.

18. World Anti-Doping Agency official website (www.wada-ama.org) accessed February 10, 2004.

19. World Anti-Doping Agency press release "WADA Executive Committee Approves the 2006 Prohibited List" Dated: September 20, 2005.

20. World Anti-Doping Agency. World Anti-Doping Agency 2004 Annual Report. Montreal, Canada, 2004 